\title{
Quantum Chaos, Irreversible Classical Dynamics and Random Matrix Theory
}

\author{
A. V. Andreev ${ }^{a, b}$, O. Agam ${ }^{a}$, B. D. Simons ${ }^{c}$, and B. L. Altshuler. ${ }^{a, b}$ \\ ${ }^{a}$ NEC Research Institute, 4 Independence Way, Princeton, NJ 08540, USA \\ ${ }^{b}$ Department of Physics, Massachusetts Institute of Technology, 77 Massachusetts Avenue, Cambridge, MA 02139, USA \\ ${ }^{c}$ Cavendish Laboratory, Madingley Road, Cambridge, CB3 OHE, UK
}

\begin{abstract}
The Bohigas-Giannoni-Schmit conjecture stating that the statistical spectral properties of systems which are chaotic in their classical limit coincide with random matrix theory is proved. For this purpose a new semiclassical field theory for individual chaotic systems is constructed in the framework of the non-linear $\sigma$-model. The low lying modes are shown to be associated with the Perron-Frobenius spectrum of the underlying irreversible classical dynamics. It is shown that the existence of a gap in the Perron-Frobenius spectrum results in a RMT behavior. Moreover, our formalism offers a way of calculating system specific corrections beyond RMT.
\end{abstract}

The theory of random matrices [1] emerged from the need to characterize complex quantum systems in which knowledge of the Hamiltonian is minimal, e.g. complex nuclei. The basic hypothesis is that the Hamiltonian may be treated as one drawn from an ensemble of random matrices with appropriate symmetries. It has been proposed by invoking the complexity of systems which have many degrees of freedom with unknown interaction coupling among them.

The study of the statistical quantum properties of systems with small number of degrees of freedom, within the framework of random matrix theory (RMT), has developed along two parallel lines. The first was by considering an ensemble of random systems such as disordered metallic grains [2]. Randomness in this case is introduced on the level of the Hamiltonian itself, e.g. as a consequence of the unknown impurity configuration. In the second approach, RMT was used in order to understand the level statistics of non-stochastic systems which are chaotic in their classical limit such as the Sinai or the stadium billiards [3]. Here "randomness" is generated by the underlying deterministic classical dynamics itself. Nevertheless, it has been conjectured [3] that "spectrum fluctuations of quantal time-reversal invariant systems whose classical analogues are strongly chaotic have the Gaussian Orthogonal Ensembles pattern".

Despite being supported by extensive numerical studies, the origin of the success of RMT as well as its domain of validity are still not completely resolved. In this letter we show that, in the semiclassical limit, this conjecture is indeed valid for systems with exponential decay of classical correlation functions in time. Moreover, the formalism which we introduce below offers a way of calculating system specific corrections beyond RMT.

So far, the main attempts to establish the relationship between non-stochastic chaotic systems and RMT, have been based on periodic orbit theory 依. Gutzwiller's trace formula expresses the semiclassical density of states as an infinite sum over the classical periodic orbits of the system. However, the number of periodic orbits is exponentially large and clearly contains information that is redundant from quantum mechanical point of view. This detailed information conceals the way of drawing a connection between the quantum behavior of chaotic systems and RMT. Indeed, the success of the periodic orbit theory approach in reproducing RMT results [5] appears to be limited.

Here we develop a new semiclassical approach in which the basic classical ingredients are not individual periodic orbits but global modes of the time evolution of the underlying classical system. It is possible to construct a field theory in which the effective action is associated with the classical flow in phase space. We argue that, the statistical quantum properties of the system are intimately related to the irreversible classical chaotic dynamics or, more precisely, to the Perron-Frobenius modes, in which a disturbance in the classical probability density of chaotic system relaxes into the ergodic distribution. These modes decay at different rates. This, in principle, enables a description of the system at levels of increasing complexity by incorporating higher and higher modes. The "zero mode" manifests the conservation of classical probability and corresponds to a uniform distribution over the energy shell. We show that RMT coincides with a description which takes into account only this mode. The deviations from the universal RMT behavior emerge from the higher modes of the evolution of the classical system.

Our approach is analogous to that of disordered systems where the diffusion modes account for the classical relaxation. However, in the field theoretic description of disordered systems [7] averaging is performed over an ensemble. Here, in contrast, in order to characterize individual systems only energy averaging will be employed.

The main result of this letter will be to establish the Bohigas-Giannoni-Schmit conjecture. To do so we will first show that quantum statistical correlators are described by a functional non-linear $\sigma$-model. Its low lying modes are identified with the Perron-Frobenius eigenmodes of the underlying classical dynamics. Then we argue that, provided classical correlation functions decay exponentially in time, there is an energy domain where the zero mode contribution governs the behavior. This follows from the fact that in such systems the PerronFrobenius spectrum has a gap. Finally we establish the relation to RMT by identifying it with the zero mode of 
the constructed field theory.

Let $\hat{H}$ define a quantum Hamiltonian whose classical counterpart, $H(\mathbf{x})$, is chaotic. Here we use the notation $\mathbf{x}=(\mathbf{q}, \mathbf{p})$ to denote a vector defined in a $2 \times d$ dimensional phase space. We restrict attention to a system in which all classical trajectories are unstable, and there are no islands of regular motion in phase space. At sufficiently high energy $E_{0}$, the mean spacing $\Delta$ between adjacent energy levels can be assumed constant and expressed through the Weyl formula,

$$
\frac{1}{\Delta}=\frac{1}{(2 \pi \hbar)^{d}} \int d^{2 d} x \delta[E-H(\mathbf{x})] .
$$

Henceforth energy and time will be measured in units of $\Delta$ and the Heisenberg time $\hbar / \Delta$ respectively.

The energy averaging is taken over an energy band containing a large number of levels $N$ such that $1 \ll$ $N \ll \epsilon_{0}=E_{0} / \Delta$. For simplicity we choose to work with Gaussian averages $(\epsilon=E / \Delta)$ :

$$
\langle\cdots\rangle_{\epsilon_{0}}=\int \frac{d \epsilon}{\sqrt{2 \pi} N} \exp \left[-\frac{\left(\epsilon-\epsilon_{0}\right)^{2}}{2 N^{2}}\right](\cdots) .
$$

The basic quantity calculated within the field theory approach is the generating function $\langle\mathcal{Z}(J)\rangle_{\epsilon_{0}}$. Any $n$ point correlation function can be obtained by taking its derivatives with respect to the various components of the source $J$. However, to keep the discussion simple, we will restrict attention to systems belonging to the unitary ensemble (i.e. those with broken T-invariance), and focus on two-point correlation functions such as the correlator of density of states, $R(s)=\langle\rho(\epsilon+s) \rho(\epsilon)\rangle_{\epsilon_{0}}-1$, where $\rho(\epsilon)=\operatorname{Tr} \delta(\epsilon-H)$. As long as $s$ is sufficiently small compared to the bandwidth $N$, the final results are independent of the particular form of energy averaging.

Following the usual approach [7], the generating function can be expressed as a field integral. Introducing the four component supervector field $\Psi^{T}(\mathbf{q})=$ $\left(\psi^{R}, \chi^{R}, \psi^{A} \chi^{A}\right)$, where $\psi$ and $\chi$ denote commuting and anti-commuting components respectively, and the superscript $A(R)$ denotes the advanced (retarded) components [7], we can define

$$
\mathcal{Z}(J)=\int \mathcal{D} \Psi e^{-\mathcal{S}[\Psi, \epsilon]},
$$

where the action is given by

$$
\mathcal{S}[\Psi, \epsilon]=i \int d^{d} q \bar{\Psi}(\mathbf{q})\left[\epsilon-\frac{s^{+}}{2} \Lambda-\hat{H}-J k \Lambda\right] \Psi(\mathbf{q}) .
$$

Here $s^{+}=s+i 0$, while $\Lambda=\operatorname{diag}(1,1,-1,-1)$ and $k=\operatorname{diag}(1,-1,1,-1)$ break the symmetry between retarded/advanced field components and supersymmetry respectively, and $\bar{\Psi}=\Psi^{\dagger} \Lambda$. The use of fermionic as well as bosonic components ensures the normalization $\mathcal{Z}(0)=1$. To evaluate the two point density correlator one can choose $J$ to be constant, then

$$
R(s)=-\left.\frac{1}{16 \pi^{2}} \Re \frac{\partial^{2}\langle\mathcal{Z}(J)\rangle_{\epsilon_{0}}}{\partial J^{2}}\right|_{J=0} .
$$

Energy averaging (2) of $\mathcal{Z}(J)$ generates a quartic interaction of the $\Psi$ fields: $\mathcal{S}(\Psi, \epsilon) \rightarrow \mathcal{S}\left(\Psi, \epsilon_{0}\right)+\mathcal{S}_{\text {i }}$, where

$$
\mathcal{S}_{\mathrm{i}}=\frac{N^{2}}{2}\left(\int d^{d} q \bar{\Psi}(\mathbf{q}) \Psi(\mathbf{q})\right)^{2} .
$$

In contrast to impurity averaging [7], energy averaging induces a non-local interaction of the $\Psi$ fields. This interaction term can be decoupled by means of HubbardStratonovich transformation, with the introduction of $4 \times 4$ supermatrix fields $Q\left(\mathbf{q}, \mathbf{q}^{\prime}\right)$ which depend on two coordinates,

$$
\exp \left(-\mathcal{S}_{\mathrm{i}}\right)=\int \mathcal{D} Q \exp \left[-\operatorname{STr}_{\mathbf{q}}\left(\frac{Q^{2}}{2}-i \mathcal{N} Q \Psi \bar{\Psi}\right)\right] .
$$

Here $\mathrm{STr}_{\mathbf{q}}$ denotes the trace operation for supermatrices, while the subscript $\mathbf{q}$ implies a further extension of the trace to include integration over all spatial variables, e.g. $\operatorname{STr}_{\mathbf{q}} Q^{2}=\int d^{d} q d^{d} q^{\prime} \operatorname{STr} Q\left(\mathbf{q}, \mathbf{q}^{\prime}\right) Q\left(\mathbf{q}^{\prime}, \mathbf{q}\right)$. Integrating over $\Psi$ we obtain:

$$
\begin{array}{r}
\langle\mathcal{Z}(J)\rangle_{\epsilon_{0}}=\int \mathcal{D} Q \exp \left[-\frac{1}{2} \operatorname{STr}_{\mathbf{q}} Q^{2}+\operatorname{STr}_{\mathbf{q}} \ln \mathcal{G}\right], \\
\mathcal{G}^{-1}(Q)=\epsilon_{0}-\frac{s^{+}}{2} \Lambda-\hat{H}-J k \Lambda-N Q .
\end{array}
$$

The derivation of Eq. (8) involved no approximation. Further progress is possible only within a saddle-point approximation, which is accurate to order $1 / N$. Varying the total action with respect to $Q$ one obtains the saddle-point equation,

$$
Q_{0} \mathcal{G}^{-1}\left(Q_{0}\right)=N
$$

where $Q_{0}$ and $\mathcal{G}\left(Q_{0}\right)$ are operators.

To understand the structure of the saddle-point manifold, in the semiclassical limit, it is useful to employ the Wigner representation of operators. Given an operator $\mathcal{O}$ as a set of matrix elements $\mathcal{O}\left(\mathbf{q}_{1}, \mathbf{q}_{2}\right)$ between two position states at $\mathbf{q}_{1}$ and $\mathbf{q}_{2}$, its Wigner representation is a function of the phase space variables $\mathbf{x}$ defined by $\mathcal{O}(\mathbf{x})=\int d^{d} q^{\prime} \exp \left(i \mathbf{p} \mathbf{q}^{\prime} / \hbar\right) \mathcal{O}\left(\mathbf{q}+\mathbf{q}^{\prime} / 2, \mathbf{q}-\mathbf{q}^{\prime} / 2\right)$. We will use the fact that, in the semiclassical limit, the Wigner transform of a product of operators is equal the product of the Wigner transformed operators, namely, $\left(\mathcal{O}_{1} \mathcal{O}_{2}\right)(\mathbf{x}) \rightarrow \mathcal{O}_{1}(\mathbf{x}) \mathcal{O}_{2}(\mathbf{x})$, where $\mathcal{O}_{1,2}(\mathbf{x})$ are smooth slowly varying functions on quantum scale.

Treating $s$ and $J$ as small compared to the bandwidth $N$, and introducing the phase space variables, $\mathbf{x}_{\|}$on the energy shell and $x_{\perp}=H$ perpendicular to the energy shell, the solution of Eq. (10) can be written in the Wigner representation as

$$
Q_{0}(\mathbf{x})=\frac{\epsilon_{0}-H}{2 N}+i\left[1-\left(\frac{\epsilon_{0}-H}{2 N}\right)^{2}\right]^{\frac{1}{2}} \Lambda .
$$


This solution is, however, not unique. In fact, the saddlepoint solutions form a degenerate manifold in superspace associated with the group of pseudo-unitary rotations. Both the integration measure and the action in Eq. (8) are invariant under the group of transformations $Q(\mathbf{x}) \rightarrow U^{-1}\left(x_{\perp}\right) Q(\mathbf{x}) U\left(x_{\perp}\right)$, where $U\left(x_{\perp}\right)$ belongs to the pseudo-unitary super-group $U(1,1 / 2)$ [8]. Thus, any matrix of the form $Q\left(x_{\perp}\right)=U^{-1}\left(x_{\perp}\right) Q_{0}\left(x_{\perp}\right) U\left(x_{\perp}\right)$ is a solution of the saddle-point equation (8).

When integrating over the fluctuations $\delta Q$, near the saddle-point manifold, one has to take into account the anisotropy of the dependence of the action on the fluctuations $\delta Q$. Those fluctuations on which the action depends strongly (massive modes) can be integrated out within a conventional saddle-point approximation. The remaining fluctuations describe the Goldstone modes of the system, and their integration must be performed exactly. The experience gained from studies of disordered systems suggests that these degrees of freedom can be parametrized by $Q(\mathbf{x})=T^{-1}(\mathbf{x}) Q_{0}\left(x_{\perp}\right) T(\mathbf{x})$. However, integration over the massive modes [9] shows that, in the limit $N \gg 1$, the only non-vanishing contribution comes from matrices $T$ which are independent of the energy $x_{\perp}$. The Goldstone modes can be therefore parametrized by

$$
Q(\mathbf{x})=T^{-1}\left(\mathbf{x}_{\|}\right) Q_{0}\left(x_{\perp}\right) T\left(\mathbf{x}_{\|}\right),
$$

where $T\left(x_{\|}\right)$belong to the coset space $U(1,1 / 2) / U(1 / 1) \otimes U(1 / 1)$ 8].

The derivation of the effective field theory can thus be obtained by: (i) Substituting Eq. (12) into Eq. (8), approximating commutators by Poisson brackets $\left[\mathcal{O}_{1}, \mathcal{O}_{2}\right](\mathbf{x}) \rightarrow i \hbar\left\{\mathcal{O}_{1}(\mathbf{x}), \mathcal{O}_{2}(\mathbf{x})\right\}$, and replacing the trace by the phase space integral, $\operatorname{Tr}_{\mathbf{q}}(\mathcal{O})=h^{-d} \int d \mathbf{x} \mathcal{O}(\mathbf{x})$ (this is the entry point of the semiclassical analysis) (ii) Expanding the logarithm to first order in $s^{+}, J$, and the Poisson bracket $\left\{H, T\left(\mathbf{x}_{\|}\right)\right\}$(higher order terms of this expansion appear at order $1 / N$ and are neglected) (iii) Performing the $x_{\perp}$ integration of the resulting action. The last step relies on the fact that within the energy band, where averaging takes place, the classical dynamics is independent of the energy. As a result we obtain the $\sigma$-model:

$$
\begin{array}{r}
\langle\mathcal{Z}(J)\rangle_{\epsilon_{0}}=\int \mathcal{D} T\left(\mathbf{x}_{\|}\right) \exp \left(-\mathcal{S}_{\text {eff }}\right), \\
\mathcal{S}_{\text {eff }}=\frac{i \pi}{h^{d}} \int d \mathbf{x}_{\|} \operatorname{STr}\left[\left(\frac{s^{+}}{2} \Lambda+J k \Lambda\right) \mathcal{Q}-i \mathcal{Q} T^{-1} \mathcal{L} T\right]
\end{array}
$$

where

$$
\mathcal{Q}=-\frac{i}{\pi N} \int d x_{\perp} T^{-1}\left(\mathbf{x}_{\|}\right) Q_{0}\left(x_{\perp}\right) T\left(\mathbf{x}_{\|}\right)=T^{-1} \Lambda T,
$$

and $\mathcal{L}$ is the dimensionless infinitesimal time evolution operator defined by the Poisson bracket

$$
\mathcal{L} \cdot=\hbar\{H, \cdot\}
$$

For a stochastic Hamiltonian, an action equivalent to Eq. (14) has been proposed recently using an argument which relies on disorder averaging in the limit of vanishing disorder [10].

To make sense of the functional integral in Eq. (13) we must identify the low lying modes of the action. In the case of impurities, the low lying degrees of freedom correspond to the eigenvalues of the diffusion operator that form a discrete spectrum. In the present case it is tempting to associate the low energy degrees of freedom with eigenmodes of the unitary (reversible) evolution operator $e^{-\mathcal{L} t}$. However this identification is incorrect.

As with any functional integral there is a need to define an appropriate regularization. For example the functional integral in Eq. (13) may be understood as the limit $a \rightarrow 0$ of a product of definite integrations over a discretized space, where $a$ denotes the discretization cell size. This admits to functions $T\left(\mathbf{x}_{\|}\right)$which are smooth and square integrable. More generally, a regularization can be obtained using a truncation of an arbitrary complete basis.

In seeking such a basis, the eigenfunctions of the classical evolution operator $\mathcal{L}$ seem to be the natural choice. However, the intricate nature of chaotic classical evolution cause these eigenfunctions to lie generally outside the Hilbert space. This can be seen from the following consideration: Chaotic dynamics of probability densities involves contraction along stable manifolds, together with stretching along unstable ones. Thus, in the course of time, an initially non-uniform distribution turns into a singular function on the unstable manifold, which in turn covers the whole energy shell densely. Therefore the eigenfunctions of $\mathcal{L}$, which require the infinite time limit, are not square integrable and their contribution to the functional integral can not be directly recovered by the discretization procedure involved in evaluating the functional integral.

Thus, in choosing a convenient basis one has to take account of the regularization. Its primary effect is to truncate the contraction along the stable manifold and thereby render the classical evolution irreversible. It is the eigenfunctions of this regularized classical evolution operator that serve as a suitable basis for the quantum mechanical correlator.

A natural way of introducing a regularization is to express the functional integral in terms of a basis of eigenfunctions of the evolution operator to which a small noise has been added such that a diffusion on the energy shell is allowed. The eigenfunctions of the resulting operator, which is no longer antihermitean, are rendered smooth along the stable manifold and square-integrable. When the strength of the noise is taken to zero - the spectrum of the resulting operator, known in the literature as the Perron-Frobenius operator, reflects intrinsic irreversible properties of the purely classical dynamics [11]. Other approaches which recover this spectrum involved a use of symbolic dynamics [12], course graining of the flow dynamics in phase space 13. and analytic continuation methods 14. We remark here that the physical spectrum of the classical operator $\mathcal{L}$ appears when it prop- 
agates smooth probability densities. Thus the matrices $T\left(\mathbf{x}_{\|}\right)$over which $\mathcal{L}$ acts are understood to be smooth. In this respect, quantum mechanics can viewed as the natural framework for calculating irreversible properties of classically chaotic systems.

Let $\left\{\gamma_{n}\right\}$ be the set of the eigenvalues of the generator, $\mathcal{L}$, of the Perron-Frobenius operator. In ergodic systems, the leading eigenvalue $\gamma_{0}=0$ is non-degenerate, and manifests the conservation of probability density. Thus any initial density relaxes, in the course of time, to the state associated with $\gamma_{0}$. If, in addition, this relaxation is exponential in time, then the Perron-Frobenius spectrum has a gap associated with the slowest decay rate. Thus, for the first nonzero eigenvalue $\gamma_{1}$ we have $\gamma_{1}^{\prime} \equiv \operatorname{Re}\left(\gamma_{1}\right)>0$. This gap sets the ergodic time scale, $\tau_{c}=1 / \gamma_{1}^{\prime}$, over which the classical dynamics relaxes to equilibrium. In the case of disordered metallic grains, it coincides with the Thouless time, while in ballistic systems or billiards it is of order of the time of flight across the system.

In the limit $s \ll \gamma_{1}^{\prime}$ the dominant contribution to Eq. (13) comes from the ergodic classical distribution, the zero-mode $\mathcal{L} T_{0}=0$. With this contribution alone, the functional integral (13) becomes definite,

$$
\langle\mathcal{Z}(J)\rangle_{\epsilon_{0}}=\int d \mathcal{Q}_{0} \exp \left(-i \frac{\pi}{2} \mathrm{~S} \operatorname{Tr}\left[\left(s^{+} \Lambda+2 J k \Lambda\right) \mathcal{Q}_{0}\right]\right)
$$

where $\mathcal{Q}_{0}=T_{0}^{-1} \Lambda T_{0}$. This expression coincides with that for RMT and leads to the Wigner-Dyson statistics [7, 15.

This result can be generalized to any $n$-point correlation function as well as to systems with T-invariance. One thus concludes that the quantum statistics of chaotic systems with exponential classical relaxation are described by RMT at energies smaller than $\gamma_{1}^{\prime}$.

RMT description is expected to hold even for certain chaotic systems where the Perron-Frobenius spectrum is gapless [3], such as the stadium or the Sinai billiards where classical correlation functions decay algebraically in time [16]. The resolvent $1 /(z-\mathcal{L})$, in this case, is expected to have cuts which reach the $\Im z$ axis. Nevertheless, we expect the RMT description to hold whenever the spectral weight of the resolvent inside the strip $0 \leq \Re z \leq 1$ (which excludes the pole at the origin, however) is much smaller than unity.

The strength of the field theoretic approach is that it encompasses a range of energy scales which go well beyond RMT. In particular, a similar procedure to that used in Ref. 17] can be employed to obtain $R(s)$ corresponding to the unitary ensemble

$$
R(s)=-\frac{1}{4 \pi^{2}} \frac{\partial^{2}}{\partial s^{2}} \ln \mathcal{D}(s)+\frac{\cos (2 \pi s)}{2 \pi^{2}} \mathcal{D}(s),
$$

where $\mathcal{D}(s)=\prod_{\mu} A_{\mu}\left(s^{2}+\gamma_{\mu}^{2}\right)^{-1}$ is the spectral determinant $\left(A_{\mu}\right.$ being regularization factors [18]) associated with the Perron-Frobenius spectrum. This confirms the conjecture made Ref. 18].

In conclusion, we have established the BohigasGiannoni-Schmit conjecture for chaotic systems in which classical correlation functions decay sufficiently fast. Moreover, the field theoretic approach allows the study of statistical characteristics of such systems on an energy scale which is much wider than that in which RMT applies. These statistics are determined by the analytic properties of the classical resolvent operator $1 /(z-\mathcal{L})$. This theory, in principle, offers a systematic controlled way of investigating quantum corrections to the leading semiclassical description.

We are grateful to D. E. Khmel'nitskii, B. A. Muzykantskii, A. M. Polyakov, D. Ruelle Ya. G. Sinai and N. Taniguchi for stimulating discussions. This work was supported in part by JSEP No. DAAL 03-89-0001 and by NSF Grant No. No. DMR 92-14480. O. A. acknowledges the support of the Rothschild Fellowship.

[1] M. L. Mehta, Random Matrices, (Academic Press, New York, 1991)

[2] L. P. Gor'kov and G. M. Eliashberg, Zh. Eksp. Teor. Fiz., 48, 1407 (1965) [JETP, 21, 940]; B. L. Altshuler and B. I. Shklovskii, JETP 64, 127 (1986).

[3] O. Bohigas, M. J. Giannoni, and C. Schmidt, Phys. Rev. Lett. 52, 1 (1984).

[4] M. C. Gutzwiller, Chaos in Classical and Quantum Mechanics (Springer, NY, 1990).

[5] M. V. Berry, Proc. Roy. Soc. London A 400, 229 (1985).

[6] J. H. Hannay and A. M. Ozorio de Almeida, J. Phys. A 17, 3429 (1984).

[7] K. B. Efetov Adv. Phys. 32, 53 (1983).

[8] M. R. Zirnbauer, Nucl. Phys. B 265 [FS 15], 375 (1986).

[9] A. V. Andreev, O. Agam, B. D. Simons, and B. L. Altshuler, in preparation.

[10] B. A. Muzykantskii, D. E. Khmel'nitskii, JETP Lett. 62, 76 (1995). In this case the equivalent action is expressed in the form of a Wess-Zumino-Witten term.

[11] P. Gaspard, G. Nicolis, A. Provata, and S. Tasaki, Phys. Rev. E. 51, 74 (1995).

[12] D. Ruelle, Statistical Mechanics, Thermodynamic Formalism (Reading, MA: Addison-Wesley, 1978).

[13] G. Nicolis and C. Nicolis, Phys. Rev. A. 38, 427 (1988).

[14] H. H. Hasegawa and D. J. Driebe, Phys. Rev. E. 50, 1781 (1994).

[15] J. J. M. Verbaarschot, H. A. Weidenmuller, M. R. Zirnbauer, Phys. Rep. 129, 367 (1985).

[16] L. A. Bunimovich, Sov. Phys. JETP 62 (4), 842 (1985).

[17] A. V. Andreev and B. L. Altshuler, Phys. Rev. Lett. 75, 902 (1995).

[18] O. Agam, B. L. Altshuler and A. V. Andreev Phys. Rev. Lett. 75, 4389 (1995). 